\title{
Nutrición de yodo en escolares de Calama, Chile: persitencia de niveles elevados de yodo urinario
}

\author{
lodine nutrition in school children of Calama city, Chile: \\ persistence of high urinary iodine levels
}

\begin{abstract}
Introduction: In the year 2000 it was determined that the median of the urinary iodine concentration (UIE) in primary school children from Calama was 2600 ug/L, a very high level which went down to $487 \mathrm{ugl} / \mathrm{L}$ in 2004, after the standards of the Food Sanitary Regulation were scaled down from $100 \mathrm{ppm}$ to $40 \mathrm{ppm}$ in the year 2000. Objective: To search the current iodine nutrition in school children of Calama, 12 years after the change in the legislation. Subjects and methods: 332 primary school children from Municipal School D-48 of the City of Calama were assessed in relation to their prevalence of goiter determined through palpation of the thyroid gland. In $22 \%$ of them (72 children), UIE was determined by spectrophotometry. 58 samples of salt for human consumption salt were randomly collected from local shops, school canteens and from the children's homes to determine their iodine concentration through the starch technique. In addition, the school children were measured and weighed to determine the adequacy of their height and Body Mass Index (BMI). Results: An 8.2\% prevalence of goiter was found; all were grade 1. The iodine concentration in the salt was $31.4 \pm 17.8$ in the 58 samples analyzed, the average met the adequate range of the current Food Sanitary Regulation (FSR) of Health Ministry of Chile. Conclusion and comments: A non significant increase in the prevalence of goiter was found, the average of UIE continue being high, possible due to an excessive intake of iodine, nevertheless average iodine concentration in the salt for human consumption is within de recommended range by WHO-UNICEF-ICCIDD (20-40 ppm). These findings prompt to search another sources of iodine intake, as an explanation of the persistent elevation of UIE in these children.

Key words: Endemic goiter, urinary iodine excretion, salt iodine concentration, nutritional status, stature adequacy.
\end{abstract}

\section{INTRODUCCIÓN}

La erradicación del bocio endémico ha sido exitosa en numerosos países, entre ellos Chile, sin embargo continúa siendo un problema de salud pública en algunos debido a dificultades en la producción, mantención y monitarización continua de la yodación de la sal (1). Chile manifestó su intención de erradicar el bocio endémico en 1959 al promulgar la ley de yodación de la sal de consumo humano. Esta medida de comprobada efectividad, se implementó sólo en 1979 agregando a la sal de consumo humano una parte de yodato de sodio o potasio por cada 10.000 partes de sal.

Antes de iniciar la yodación de la sal la prevalencia de bocio en el país, se conocía sólo por investigaciones parciales.
Santiago Muzzo B. (1)

Eduardo Pretell (2)

Francisco Bueno N. (.3)

Carolina Muñoz M. (4)

(1) Dirección de Investigación, Facultad de Medicina, Universidad Diego Portales, Santiago, Chile.

(2) Coordinador Regional para America del ICCIDD (International Council for Control of lodine Deficience Disorders), Lima, Perú. (3) Servicio de Obstetricia, Hospital de Calama, Calama, Chile. (4) Servicio de Nutrición. Hospital de Calama, Calama, Chile.

Dirigir la correspondencia a:

Dr. Dirección de Investigación Facultad de Medicina Universidad Diego Portales Grajales 1746 Santiago, Chile Fono: 2 6768702

E-mail: santiago.muzzo@udp.cl

Este trabajo fue recibido el 7 de Enero de 2013 y aceptado para ser publicado el 7 de Febrero de 2013.

Las publicaciones indicaban un problema moderado de bocio endémico, sin casos de cretinismo endémico (2 3); mas serio en grupos cerrados como Pedregoso (61\% ), Lonquimay (31\%) (4) Puente Alto, Pirque y San José de Maipo (25\%) (5).

La primera información representativa de la prevalencia de bocio endémico en escolares de educación básica fue publicada por Muzzo y colaboradores quienes detectaron en 1982 en la Región Metropolitana una prevalencia de 18,8\% (6). Estudios posteriores realizados en zonas censorias: norte (Calama), centro (Santiago), sur (Temuco) y extremo sur (Punta Arenas) han entregado información periódica de la nutrición de yodo, lo que ha permitido una vigilancia del problema. En 1986 la prevalencia de bocio evaluada por palpación del 
cuello en escolares de Santiago y Temuco fue de 7,6\% (7-10).

Entre los años 1991 y 1995, estudios realizados en escolares de educación básica de 4 zonas censorias: Santiago, Temuco, Punta Arenas y Calama, mostraron una tendencia a la disminución de la prevalencia de bocio, con excreción promedio de yodo urinario elevada y en ascenso durante los últimos años (11). El control del contenido de yodo de la sal de los locales de expendio de cada localidad, era adecuada a la legislación vigente.

De acuerdo a estos resultados el Ministerio de Salud indicó la disminución de la yodación de la sal de 100 a 40 ppm, la que se incorporó al nuevo Reglamento Sanitario de los Alimentos (RSA) en Enero de 2000. Al año siguiente una nueva evaluación realizada en las 4 zonas censorias, mostró que la prevalencia de bocio continuaba baja y que a pesar que la yodación de la sal era adecuada, no se había producido el descenso esperado en las yodurias, mas aún detectándose mas altas en Calama (12). Sin embargo, el año 2004 se habían normalizado las yodurias en 3 zonas censorias (Santiago, Temuco y Punta Arenas), mientras que en Calama habían descendido sólo hasta a $487 \mathrm{ug} / \mathrm{L}$.

La mantención de una baja prevalencia de bocio en los escolares de estas zonas censorias durante los últimos 10 años, indica que el bocio endémico se ha solucionado, sin embargo, la vigilancia de la nutrición de yodo debe continuar con el fin de detectar cambios en la prevalencia de bocio y los posibles efectos secundarios de la ingesta excesiva de yodo registrada en nuestro país.

Nos interesó estudiar el estado actual de la nutrición de yodo en el escolar de educación básica de Calama y compararla con la presentaron en una muestra tomada el año 2006.

\section{SUJETOS Y MÉTODO}

Esta investigación fue aprobada por el Comité de Etica de la Facultad de Medicina de la Universidad Diego Portales. Previo a la toma de la muestra, todos los estudiantes o sus apoderados debieron firmar un consentimiento informado.

Se evaluaron 339 escolares de ambos sexos, de educación básica de la escuela municipal D-48 de la ciudad de Calama, a los que se les determinó el grado de bocio por palpación de la glándula tiroidea de acuerdo a la clasificación OMS modificada por Pérez (13). A todos los escolares examinados se les determinó el peso en una balanza de pesas (Seca) con una precisión de $0,1 \mathrm{~K}$ y la talla en un cartabón adosado a la pesa con una precisión de $0,1 \mathrm{~cm}$. Las niñas se pesaron y midieron con ropa liviana y descalzas y los varones sin camisa y descalzos. Con el peso y la talla se calculó el Indice de Masa Corporal (IMC) (peso/talla2). La adecuación del estado nutricional se clasificó como déficit ponderal cuando el IMC era menor del percentilo 10; eutrofia entre los percentilos 10 y 85; riesgo de obesidad entre el percentilo 85 y 95 y obesidad cuando el IMC era mayor del percentilo 95 de acuerdo a la edad y sexo. La estatura se clasificó como baja cuando estaba bajo el percentilo 5; normal entre el percentilo 5 y 95 y talla alta sobre el percentilo 95 de acuerdo a la edad y sexo de las tablas del NCHS-CDC de la OMS.

Aleatoriamente al 25\% de los escolares encuestados (en total 72) se les solicitó una muestra de orina matinal para determinar en ella la concentración de yodo urinario mediante espectrofotometría (14). En la ciudad de Calama se seleccionaron aleatoriamente 58 muestras de sal de consumo humano de diferentes marcas comerciales en los supermercados locales, en los comedores del colegio y en los hogares de los escolares encuestados, a las que se determinó la concentración de yodo mediante la técnica de almidón (15). El análisis estadístico de los datos se realizó con el programa SPSS v18.0. La normalidad de las variables cuantitativas se determinó por medio de la prueba de Kolmogorov-Smirnov. La diferencia de promedios en variables continuas se realizó por medio de la T de Student; la comparación de proporciones por la prueba de Chi2 y Mann Whitney $U$ test. Para las muestras que no presentaban normalidad se usó el test no paramétrico de Kruscal Wallis y el test de Bonferroni. Para los análisis de correlación (asociación) su utilizó la prueba de correlación por rangos de Spearman. Se fijo el nivel de significancia en 0,05.

\section{RESULTADOS}

Se encuestaron y examinaron 339 alumnos de ambos sexos de educación básica (primero a octavo año) de la Escuela municipal D-48 de la ciudad de Calama, de los cuales 51.5\% eran varones. Su rango de edad era de 6 años 4 meses a 16 años 3 meses.

Las 313 determinaciones antropométricas efectuadas a escolares de ambos sexos demostraron que el 6,1\% tenía déficit de peso, 51,4\% eran eutróficos; 20,1\% tenían sobrepeso y 22,4 eran obesos ( $42,5 \%$ con exceso de peso). No existió una diferencia significativa entre la calidad del estado nutricional de los varones y las mujeres $(p=0.102)$ (tabla 1). En cuanto a la calidad estatural de los escolares, el $6,4 \%$ tenía déficit estatural, 90,4\% estatura normal y sólo el 3,2\% tenía talla alta, no existiendo una diferencia significativa en la calidad estatural entre varones y mujeres $((p=0.913)(91,1$ vs $89,5 \%$ ) (tabla 2 ).

Al palpar la glándula tiroidea de 332 escolares de ambos sexos, se detectó una prevalencia total de bocio de 8,2\%, todos ellos de grado I (tabla 3). Los casos de bocio fueron levemente mas frecuentes en las mujeres, sin que esta diferencia alcanzara una significación estadística. El 5,1\% de los bocios detectados

TABLA 1

Adecuación nutricional de los escolares de la escuela D-48 de Calama, según sexo.

\begin{tabular}{|c|c|c|c|c|c|c|c|c|c|c|}
\hline IMC & \multicolumn{2}{|c|}{$<\mathrm{P} 10$} & \multicolumn{2}{|c|}{ P10-85 } & \multicolumn{2}{|c|}{ P 85-95 } & \multicolumn{2}{|c|}{ > P 95} & \multicolumn{2}{|c|}{ TOTALES } \\
\hline SEXO & $\mathrm{N}$ & $\%$ & $\mathrm{~N}$ & $\%$ & $\mathrm{~N}$ & $\%$ & $\mathrm{~N}$ & $\%$ & $\mathrm{~N}$ & $\%$ \\
\hline VARONES & 15 & 8.9 & 80 & 47.3 & 35 & 20.7 & 39 & 23.1 & 169 & 100.0 \\
\hline MUJERES & 4 & 2.8 & 81 & 56.3 & 28 & 19.4 & 31 & 21.5 & 144 & 100.0 \\
\hline TOTALES & 19 & 6.1 & 161 & 51.4 & 63 & 20.1 & 70 & 22.4 & 313 & 100.0 \\
\hline
\end{tabular}

Pearson Chi2 (3 g.l.) $=6.2144 \mathrm{P}=0.102$ 
en escolares tenían una consistencia aumentada de la glándula a la palpación, un escolar (0.3\%) presentó 1 nódulo tiroideo y otro mas de 1 nódulo $(0,3 \%)$.

La concentración promedio de yodo en orina de 72 escolares seleccionados al azar de la muestra total de escolares fue $468.8 \pm 101,7 \mathrm{ug} / \mathrm{L}$, con una mediana de $448 \mathrm{ug} / \mathrm{L}$, un mínimo de 335 y máximo de 826 ugl/L, no detectando diferencias en su concentración por sexo (tabla 4).

En esta localidad la concentración promedio de yodo de las muestras de sal analizadas fue 31,4 $\pm 17,8 \mathrm{ugl} / \mathrm{g}$ de sal, las cuales estuvieron dentro del rango de aceptación de la exigencia actual del RSA y del rango recomendado por la OMS-UNICEF-ICCIDD (20 a 60 ppm), estando el promedio de sólo una marca de las sales analizadas bajo el rango de aceptación (tabla 5).

La tabla 5 presenta la concentración de yodo de las principales sales expendidas en la ciudad de Calama y en uso en el colegio y en los hogares de los escolares, encontrando una diferencia significativa por el test no paramétrico de Kruscal Wallis entre diferentes tipos de sales, entre ellas Biosal y Sal Lobos con respecto a Merkat $(p<0,01)$ analizadas por el test de Bonferroni.

\section{DISCUSIÓN}

Las mediciones antropométricas efectuados a escolares de educación básica de este estudio mostraron una alta prevalecía de sobrepeso y obesidad (20,1 y 22,4\% respectivamente) totalizando un $42,5 \%$ con exceso de peso, sin diferencias estadísticas entre varones y mujeres, lo que concuerda con la alta prevalencia en nuestro país, la cual ha aumentado muy rápidamente durante las últimas décadas $(16,17)$. La Encuesta Nacional de Salud realizada en Chile el año 2010 (18) mostró en sujetos mayores de 15 años una prevalencia de sobrepeso de 45,3 y $33,6 \%$ en varones y mujeres; de obesidad 19,2 y $30.7 \%$ en varones y mujeres y de obesidad mórbida 1,2 y $3,3 \%$ en varones y mujeres, respectivamente. Olivares y cols (19) el año 2007 encontraron en 358 niñas pertenecientes a escuelas municipales de la Región Metropolitana de Chile que cursaban tercero a octavo básico una prevalencía de sobrepeso de $18,8 \%$ y de obesidad de 15\%. El año 2006 la Junta Nacional de Jardines infantiles (JUNJI) de Chile (JUNJI) detectó en niños de 2 a 5 años una prevalencia de sobrepeso de 22,7\% y de obesidad de $10.3 \%$ (20). La Junta Nacional de Auxilio Escolar y Becas (JUNAEB) de Chile el año 2006 encontró en escolares de primero básico del país una prevalencia de obesidad de 19,4\% (21). El presente estudio que fue tomado el año 2011 muestra comparativamente con estos otros estudios que el exceso de peso en estos escolares de Calama es alarmante y merece acciones específicas para disminuirla.

Respecto a la calidad estatural de estos escolares la antropometría mostró que sólo un 6,4\% tenía un retraso estatural lo que muestra la extraordinaria mejoría de la estatura del

TABLA 2

Adecuación estatural de escolares de la escuela D-48 de Calama, según sexo.

\begin{tabular}{|c|c|c|c|c|c|c|c|c|}
\hline \multirow[b]{2}{*}{ SEXO } & \multicolumn{2}{|c|}{$<$ P5 } & \multicolumn{2}{|c|}{ P 5-95 } & \multicolumn{2}{|c|}{ > P 95} & \multicolumn{2}{|c|}{ TOTALES } \\
\hline & $\mathrm{N}$ & $\%$ & $\mathrm{~N}$ & $\%$ & $\mathrm{~N}$ & $\%$ & $\mathrm{~N}$ & $\%$ \\
\hline VARONES & 10 & 5.9 & 154 & 91.1 & 5 & 3.0 & 169 & 100.0 \\
\hline MUJERES & 10 & 7.0 & 129 & 89.5 & 5 & 3.5 & 144 & 100.0 \\
\hline TOTALES & 20 & 6.4 & 283 & 90.4 & 10 & 3.2 & 313 & 100.0 \\
\hline
\end{tabular}

Pearson Chi2 (2 g.I.) $=0.1826 \mathrm{P}=0.913$

TABLA 3

Grado de bocio en los escolares examinados.

\begin{tabular}{lccc}
\hline GRADO & $\mathrm{n}$ & $\%$ & $\%$ acumulado \\
Sin bocio & 304 & 91.8 & 91.8 \\
1A & 22 & 6.7 & 98.5 \\
1B & 5 & 1.5 & 100.0 \\
Total & 331 & 100.0 & \\
\hline
\end{tabular}

TABLA 4

Concentración de yodo en orina (ug/L) de escolares examinados.

\begin{tabular}{cccc}
\hline Media & D.E. & Mínimo & Máximo \\
468.8 & 101.7 & 335 & 826 \\
\hline
\end{tabular}


niño chileno comparada con la que tenía hace 30 años atrás (22) cuando existía un retraso estatural en $40 \%$ escolares de ambos sexos, recuperación que se debería especialmente a que la desnutrición infantil fue erradicada en nuestro país.

La concentración de yodo urinario, de yodo en sal de consumo humano y la prevalencia de bocio, se consideran las mediciones necesarias para conocer la nutrición de yodo de una población. Las determinaciones de la concentración de yodo efectuadas en sal en la comunidad estudiada demostraron, que salvo una excepción, los promedios de los diferentes tipos de sales expendidas en la ciudad de Calama se encontraban con un nivel adecuado de yodo de acuerdo a la exigencia actual del RSA y a lo recomendado por WHOUNICEF-ICCIDD.

Han transcurrido 12 años desde que el Reglamento Sanitario de los Alimentos (RSA) de Chile indicó disminuir la yodación de la sal de 100 a 40 ppm. Las concentraciones de yodo urinario detectadas en escolares de este estudio han confirmado que la medida fue efectiva, ya que a los 3 años del cambio del RSA se normalizaron las yodurias de los escolares de las zonas censorias de Santiago, Temuco y Punta Arenas, mientras que en los escolares de Calama se observó un notorio descenso de las yodurias (bajaron de 2600 a $487 \mathrm{ug} / \mathrm{L}$ ) $(23,24)$. Las mediciones de las yodurias en los escolares de este estudio demostraron que aun permanecen en un promedio elevado (se recomienda sea menor de $300 \mathrm{ug} / \mathrm{L}$ ). No hemos detectado exceso de yodo en la sal de consumo humano, ni en el contenido de yodo de algunos alimentos analizados (pan, pollo, carne, leche fluida) ni en el agua potable de esta ciudad (datos por publicar) que explicaran una contaminación de alimentos con yodo en esta comunidad.

Gomo y cols encontraron yodurias significativamente mas altas en una zona rural comparada con una urbana atribuyéndolo a fuentes dietarias alternativas no identificadas (25). Si bien, esta podría ser una causa de la permanente elevación de las yodurias en escolares de Calama, también sería de interés descartar alguna fuente inhalatoria de yodo por la cercanía de la ciudad de Calama a zonas mineras de extracción de cobre. La excreción de yodo urinaria de escolares de Calama detectada durante los últimos 19 años en nuestro país indicaría que esta población ha estado ingiriendo crónicamente un exceso de yodo (11) Este exceso de yodo podría estar produciendo alteraciones de la glándula tiroidea que podrían dar cuenta de un posible aumento de prevalencia de tiroiditis autoinmunes, tal como se ha observado en otros lugares (26-29).
La prevalencia de bocio en escolares de enseñanza básica del presente estudio no mostró variaciones con respecto a lo detectado por el mismo investigador en mediciones anteriores. Es importante destacar que la prevalencia de bocio detectada en esta investigación se considera levemente elevada de acuerdo a la descripción de bocio endémico de la OMS (prevalencia de bocio mayor del 5\% en escolares de educación básica de una comunidad). Sin embargo, en estudio anterior detectamos que esta prevalencia de bocio por palpación de la glándula tiroidea corresponde a una de $5 \%$ detectada en los mismos escolares mediante un estudio ecográfico de la glándula tiroidea (30).

Esta ingesta excesiva de yodo debería haberse normalizado luego de la modificación del RSA el año 2000, situación que se ha dado en las zonas censorias controladas, con la excepción de Calama. La persistencia de valores significativamente mas altos de yodurias en Calama comparado con las otras localidades estudiadas sugiere que en este lugar existirían otras fuentes de yodo además del yodo suplementado en la sal y presente en otros alimentos, cuya naturaleza desconocemos y que consideramos de alto interés investigar.

También sería de mucho interés estudiar el nivel de yodo que reciben los lactantes con alimentación exclusiva al pecho, por las posibles consecuencias que pudiera tener en ellos la ingesta excesiva de yodo durante un período de tiempo prolongado.

\section{CONCLUSIONES}

La prevalencia de bocio aun permanece discretamente elevada en escolares de Calama, si bien, su determinación ecográfica podría estar normal.

La excreción urinaria de yodo en escolares de educación básica de una escuela municipal de Calama aun persiste alta, lo que indicaría la necesidad de estudiar su causa para normalizarla y así evitar consecuencias posteriores.

La concentración de yodo en sales de consumo humano recolectadas en Calama estarían dentro de lo recomendado por OMS-UNICEF-ICCIDD

Las prevalencia de sobrepeso y obesidad de estos escolares es muy alta, por lo que se deberían tomar medidas adecuadas para disminuirla.

\section{RESUMEN}

Introducción: El año 2000 se determinó que la mediana de la concentración urinaria de yodo (CUI) en escolares de

\section{TABLA 5}

Concentración de yodo (ug I/g de sal) en sales de consumo humano de la ciudad de Calama.

\begin{tabular}{|c|c|c|c|c|c|}
\hline TIPO SAL & PROMEDIO & D.E. & MINIMA & MAXIMA & $\mathrm{n}$ \\
\hline Líder & 30.0 & 14.8 & 20.0 & 47.0 & 3 \\
\hline Merkat & 15.3 & 6.9 & 5.0 & 23.0 & 6 \\
\hline Mi tierra & 43.0 & 7.1 & 38.0 & 48.0 & 2 \\
\hline Sal Lobos (todos los tipos) & 30.1 & 20.0 & 3.0 & 92.0 & 30 \\
\hline Venus & 29.4 & 5.9 & 23.0 & 39.0 & 7 \\
\hline Biosal albaca & 59.5 & 9.2 & 53.0 & 66.0 & 2 \\
\hline Biosal finas hierbas & 43.8 & 13.5 & 22.0 & 57.0 & 6 \\
\hline Biosal merken & 30.5 & 2.1 & 29.0 & 32.0 & 2 \\
\hline Totales & 34.1 & 17.8 & 3.0 & 92 & 58 \\
\hline
\end{tabular}


educación básica de Calama estaba en 2600 ug/L, valor muy elevado, el que descendió a 487 ug/L el año 2004 después de reducir los niveles oficiales de yodación de la sal de 100 ppm a 40 ppm el año 2000. Objetivo: Investigar la nutrición de yodo en escolares de Calama, 12 años después del cambio de legislación. Sujetos y métodos: 332 escolares de ambos sexos de una escuela municipal de educación básica de Calama fueron evaluados sobre la prevalencia de bocio mediante la palpación de la glándula tiroides; al 22\% de los mismos (72) se les evaluó la nutrición de yodo mediante la determinación de CUI en muestras casuales de orina por espectrofotometría. Se recolectaron 58 muestras de sal de consumo humano, seleccionadas aleatoriamente en locales comerciales, comedores de la escuela y en los hogares de los escolares, para determinar su contenido de yodo por la técnica de almidón. Adicionalmente, en todos los escolares se registró peso, talla e IMC. Resultados: Se encontró una prevalencia de bocio de $8.2 \%$, todos del grado 1. La mediana de la concentración urinaria de yodo fue $448 \mathrm{ug} / \mathrm{L}$. La concentración de yodo en la sal fue 31,4 417.8 ppm, valor dentro de los estándares oficiales establecidos en el Reglamento Sanitario de los Alimentos (RSA) del Ministerio de Salud de Chile. Comentarios y conclusiones: Los resultados muestran un leve aumento en la prevalencia de bocio y que persiste una alta CUI en escolares de Calama, probablemente debido a una excesiva ingesta de yodo, no obstante que el promedio de contenido de yodo en la sal para consumo humano estuvo dentro del rango recomendado por OMS-UNICEF-ICCIDD (20-40 ppm). Estos hallazgos plantean la necesidad de investigar otras probables fuentes de ingesta de yodo, como causa del exceso de yodo que persiste en Calama.

Palabras clave: bocio endémico, excreción urinaria de yodo, concentración de yodo en sal, estado nutricional, adecuación estatural.

Agradecimientos: Esta investigación fue financiada por el ICCIDD, entidad que no tuvo participación en el análisis de los resultados ni en su publicación. Se agradece la colaboración del Hospital de Calama, en especial a su Servicio de Nutrición por las facilidades aportadas para la toma y mantención refrigerada de las muestras. Un agradecimiento especial al Director de la Escuela D -48 de Calama Sr. Eduardo Ilaja y a sus profesores; a las nutricionistas Paula Gutierrez, Tamara Gallardo, Yeimy Argandoña y Lilian Orella por el apoyo en la recolección de la información.

\section{BIBLIOGRAFÍA}

1. ICCIDD/UNICEF/WHO. Global prevalence of iodine deficiency disorders. MDIS Working paper \#1.Micronutrient Information System, Ginebra, Organización Mundial de la Salud, 1993.

2. Donoso F, Jadresic A, López E, Garcia de los Ríos M, Concha J, Espejo M, González H, Valenzuela J, Wainstein E. Encuesta de bocio en escolares de la provincia de Santiago y consideraciones sobre el problema de bocio endémico. Rev Med Chile 1955; 83 (Supl).

3. Donoso F, Lennon H, Canessa M, Vallejos J, Aldunate $G$. Encuesta de bocio en escolares de la provincia de Linares y Ñuble. Rev Méd Chil 1959; 87:717.

4. Nagel $R$, Etcheverry R, Guzmán C, Hille A, Barzelatto J, Covarrubias E. Encuesta de bocio endémico en la población Mapuche y en algunos colegios de primera enseñanza de las provincias de Cautín y Malleco. Rev Méd Chil; 1962, 90:616-8.

5. Domínguez M, Quesney F, Michaud P, Maggiolo G, Ugarte
JM. La prevalencia del bocio en escolares de las comunas de Puente Alto, Pirque, y San José de Maipo: estudio clínico epidemiológico. Rev Méd Chil 1974;102:633.

6. Muzzo S, Leiva L, Zvaighaft A. Características del bocio endémico en el escolar de la Región Metropolitana de Chile. Rev Chil Nutr 1985; 13 (3):143-7.

7. Muzzo S, Pozo M, Zvaighaft A, Rodewald A.M., Biolley $E$, y Leiva L. Características actuales del bocio endémico en escolares de dos zonas censoras de Chile. Rev Chil Nutr 1989; 17:60-5.

8. Leiva L, Pozo M, Biolley E., Muzzo S. Excreción de yodo urinario y función tiroidea de escolares de Santiago y Temuco. Rev Chil Nutr 1992; 20:38-42.

9. Pozo M, Rodewald A.M, Biolley E, Zvaighaft, Leiva L., Muzzo S. Prevalencia de bocio endémico en escolares del centro y sur de Chile. Rev Chil Ped 1989; 60:359-62.

10. Muzzo S, Burgueño M, Carvajal F, Moreno R, Leiva L. Características actuales de bocio endémico en 3 zonas censorias de Chile. Arch Latinoam Nutr 1994; 44: 82-6.

11. Muzzo S, Burgueño M, Carvajal F, Biolley E, Avendaño $M$, Vargas $A$, Leiva $L$. Nutrición de yodo en escolares de 4 zonas censoras de Chile. Rev Med Chil 1997,125:1299-304.

12. Muzzo S, Ramirez I, Carvajal F, Biolley E, Leiva L. Nutrición de yodo en escolares de cuatro zonas de Chile en el año 2001. Rev Méd Chil 2003; 1391-8.

13. Towards the eradication of endemic goiter, cretinism and iodine deficiency, Eds: Dunn, Prettell, Daza, Viteri. PAHO cientific publication 1986; $p 502$.

14. Dunn J, Crutchfield H, Gutenkunst R, Dunn A. Methods for measuring iodine in urine. International Council for Control of lodine Deficiency Disorders. ICCIDD / UNICEF/ WHO Publicación ISBN 90-70785. 1993; pp12-19.

15. Pineda O., Dary O., Morales S. Método de campo para la determinación de yodato de sal. Sección de bioquímica nutricional. Instituto de Nutrición de Centroamérica y Panamá (INCAP). Documento Interno, 1981.

16. Kain J., Uauy R., Lira L., Taibo M., Espejo F., Albala C. Evoluación del estado nutricional de escolares chilenos de 6 años (1987-2003. Rev Med Chil 2005;133:1013-20.

17. Muzzo S, Burrows R, Cordero J, Ramírez I. "Trends in nutritional status and stature among school-age children in Chile". Nutrition 2004;20(10): 867-72.

18. Encuesta Nacional de Salud ENS 2009.2010. Ministerio de Salud. Chile. www.redsalud.gov.cl.

19. Olivares S., Bustos N., Lera L., Zelada ME. Estado nutricional, consumo de alimentos y actividad física en escolares mujeres de diferente nivel socioeconómico. Rev Méd Chil 2007;135:71-8.

20. Junta Nacional de Jardines Infantiles (JUNJI). Chile. www. junji.gob.c.

21. Junta Nacional de Auxilio Escolar y Becas. Chile. www. junaeb.cl

22. Muzzo S, Leiva L, y Zvaighaft A. Calidad de la estatura y del estado nutritivo de escolares de la Región Metropolitana de Chile. Rev Chil Nutr 1984;12 (3): 185 -9.

23. Muzzo S, Ramírez I, Carvajal F, Biolley E, Leiva L. Nutrición de yodo en escolares de cuatro zonas de Chile en el año 2001. Rev Méd Chil 2003; 131(12): 1391-8.

24. Muzzo S, Leiva L, Ramírez I, Carvajal F, Biolley E. Nutrición de yodo en escolares de una zona con alta ingesta de yodo (Calama) comparada con zona de ingesta normal (Punta Arenas). Rev Chil Nutr 2005; 32(1): 28-36.

25. Gomo Z, Allain T, Matenga J, Ndemere B, Wilson A, Urdal $P$ Urinary iodine concentrationsand thyroid function in 
adult Zimbabweans during a period of transition in iodine status. Am J Clin Nutr 1999;70:888-91.

26. Wong GWK., Lam CWK., Kwok MY., Mak TW., Ahuja AT., Chow CC, Tang W. Childhood goiter and urinary iodine excretion in Hong Kong. Eur J Pediatr 1998; 157:8-12.

27. Lauberg P., Pedersen KM., Vestergaard H., Sigurdsoson G. High incidence of multinodular toxic goitre in elderly population in low iodine intake area vs. high incidence of Grave's disease in the young in high iodine intake area: comparative surveys of thyrotoxicosis epidemilogy in East-Jutland Denmark and Iceland. Intern Med 1991; 229: 415-20.

28. Vagenakis AG., Wang CA., Burger A., Maloof F., Braverman
LE., Ingbar SH. lodine-induced thyrotoxicosis in Boston. N Engl J Med 1972; 287: 523-7.

29. Tsatsoulis A., Johnson EO., Andricula M., Kalogera C., Sverna E., Spyroy P., Seferiadis K., Tsolas O. Thyroid autoimmunity is associated with higher urinary iodine concentrations in an iodine-deficient area of Northwestern Greece. Thyroid 1999;9:279-83.

30. Pretell E.A, Delange F, Hostelek V, Carigliano S, Barreda L, Higa AM, Altschuler N, Barrogán D, Cevallos J.L, González O, Jara J.A, Medeiros-Neto G, Montes J.A, Muzzo S, Pacheco V.M, Cordero L. "Iodine nutrition improves in Latin America". Thyroid 2004;14(8):590-9. 